Commentary/Vallortigara \& Rogers: Survival with an asymmetrical brain

\section{Cerebral asymmetry: From survival strategies to social behaviour}

\author{
Jechil Sieratzki ${ }^{\mathrm{a}}$ and Bencie Woll \\ a Human Communication Science, University College London, London WC1N \\ 1PF, United Kingdom; ${ }^{\mathrm{b}}$ Language and Communication Science, City \\ University London, London EC1V OHB, United Kingdom. \\ sieratzki@vff.uni-frankfurt.de b.woll@city.ac.uk \\ http://www.city.ac.uk/lcs/deafstud_resgroup_research.htm
}

Abstract: We describe a possible link between coordinated lateralised group behaviour serving species survival in lower vertebrates and a striking lateralisation phenomenon found in human social behaviour: the universal preference for cradling a young infant on the left side. Our exploration offers a different perspective on the role of cerebral asymmetry for the survival of both the individual and the species.

Vallortigara \& Rogers (V\&R) argue that the alignment of the direction of behavioural asymmetries at the population level serves species survival by enabling individually asymmetrical organisms to coordinate their behaviour with the behaviour of other asymmetrical organisms of the same (or different) species. Drawing most of their evidence from coordinated anti-predatory behaviour in lower vertebrates, they also refer to lateralisation phenomena in the social, emotional, and communicative behaviour of higher species, including primates and humans, although without placing these behaviour patterns under the evolutionary umbrella of survival mechanisms.

We suggest a link between the lateralisation phenomena at the group level, which are the target of $\mathrm{V} \& \mathrm{R}$, and a lateralisation phenomenon found in human social behaviour at a one-to-one level: the universal preference of mothers and fathers (about 80\%), but not of males without children, to cradle a young infant on the left side, which is seen across cultures and in most artistic representations of mother-infant pairs (de Chateau 1987; Sieratzki \& Woll 1996). The split in the population between left and right cradling mirrors V\&R's data for lateralisation phenomena in lower vertebrates.

Conventional wisdom connects the use of the left arm for cradling an infant to right-handedness. However, this connection is inconsistent: the majority of sinistral mothers cradle on the left, and a substantial minority of dextral mothers cradle on their right side (Salk 1973). Close contact with the soothing maternal heartbeat has been proposed as an alternative explanation (Salk 1973); although intuitively appealing, the cardiac connection cannot account for the $20 \%$ minority of mothers who cradle on their right side (Sieratzki \& Woll 1996).

In previous work (Sieratzki \& Woll 1996), we have looked at the left cradling bias from the perspective of the role of the right hemisphere in the mother-infant relationship. After the trauma of birth the infant needs reassurance, and the mother wants nothing more than to provide this. To create the link, the mother offers her feelings through touch, gestures, facial expressions, and sounds. The mother's voice composes a melody with no or little lexical content, which shows a remarkable similarity in tune across cultures. This protolanguage is tuned to the infant's needs and responses; it is the emotional "heartbeat" that the infant seeks. All this originates from a deep-seated maternal instinct; even deaf mothers vocalise to young deaf infants (Sieratzki \& Woll 2004).

As recognised long ago by Hughlings Jackson, the right hemisphere controls intonation and affective intent of speech, that is, prosody; and this has been documented not only in $96 \%$ of righthanded people but also in more than $70 \%$ of left-handed people. A substantial body of dichotic listening experiments with subjects ranging from neonates to adults have shown significant differences in accuracy and speed of response to left- and right-ear stimuli: whereas the right ear is better for recognising structural as- 
pects of speech, the left ear is superior for recognising melodic aspects of language - in particular, affective intonation (Bryden et al. 1991).

Similarly, the right hemisphere/left visual field shows a specialisation for socio-affective signals, and this is specifically notable for a mother's recognition of expressions of distress on an infant's face (Best et al. 1994). Our own studies of blind mothers have provided evidence that the emotional impact of touch, the most basic and inherently reciprocal mode of interaction, is also more direct and immediate if an infant is held to the left side of the body (Sieratzki \& Woll 2003).

Based on the well-recognised role of the right hemisphere for perception and processing of socio-affective signals, we had theorised that the left-cradling bias was related to an advantage for mother-infant communication, which is relevant for all sensory modalities: the lullaby would not sound the same or feel the same with the baby on the other side.

This hypothesis has been confirmed by Reissland (2000) who has shown statistically significant correlations between the pitch patterns of maternal vocalisations and cradling laterality. Left cradling is associated with lower-pitch, calming, and comforting sounds; right-cradling is more often associated with higher-pitch, attention-arousing, and controlling maternal vocalisations. Depressed or insecure-anxious mothers tend to speak with a higher mean pitch and to show a higher rate of right-cradling (Reissland et al. 2003).

What does this have to do with the importance of an asymmetrical brain for the survival of the species - the topic of this target article by V\&R?

Studies of posttraumatic stress disorder have shown that severe trauma may profoundly disturb the neurophysiological balance between the left and right hemispheres, leading to the loss of normal adult bonding behaviour or failure of children to develop critical social skills. The neurophysiologist J. P. Henry (1997) has proposed that "the left and right hemispheres subserve different emotional sets that correspond to 'control' and 'appraisal,' i.e., very approximately to the self and species preservative behavioural complexes, respectively." The role that we propose for the right hemisphere in mother-infant interaction may be founded in a fundamental specialisation of this hemisphere for behavioural complexes that serve the survival of the infant and thereby of the species as a whole.

The study of the lateralisation of mother-infant interaction provides insight into a fundamental aspect of hemispheric asymmetry: its role for the survival of both the individual and the species (Sieratzki \& Woll 2002). If parental behaviour were only righthemisphere determined, just subserving infant- or species-preservative interest, the survival of the individual in the environment would not be secured. If, on the other hand, behaviour were only left-hemisphere determined and directed towards self-interest, the individual would have no reason for procreation: offspring do not offer immediate benefits in terms of survival.

During the course of evolution, the cerebral hemispheres developed different aptitudes for different tasks: sequential versus spatial and detailed versus global processing, and control versus. adaptation behaviour. The connection between these properties and lateralisation phenomena in the relation of individuals to their environment remains an intriguing question. 